\title{
Construção e Validação de Tapete de Pressão de Baixo Custo para Interação Humano-Computador
}

\author{
Thaysa Barros, João Marcelo Teixeira \\ Departamento de Eletrônica e Sistemas \\ Universidade Federal de Pernambuco \\ Recife, Brasil \\ thaysapryscilla@gmail.com \\ jmxnt@cin.ufpe.br
}

\author{
Alana E. Da Gama \\ Departamento de Engenharia Biomédica \\ Universidade Federal de Pernambuco \\ Recife, Brasil \\ aefg@cin.ufpe.br
}

\author{
Walter F. M. Correia \\ Departamento de Design \\ Universidade Federal de Pernambuco \\ Recife, Brasil \\ wfmc10@gmail.com
}

\begin{abstract}
Resumo-Diferentes tipos de sensores e tecnologias vêm sendo explorados para permitir a Interação Humano-Computador. A busca por sensores que permitam uma interação natural, sem necessidade de conexões afixadas ao corpo, ou de aprendizagem de comandos específicos é crescente. Nesse contexto, este trabalho apresenta uma tecnologia de aquisição de entrada para sistemas computacionais através da pressão, mais especificamente sensores de pressão em forma de tapetes. $O$ objetivo deste trabalho é apresentar o desenvolvimento e a validação do protótipo de um sistema de monitoramento de pressão capaz de provar conceitualmente uma alternativa de baixo custo de tapete de sensores de uso genérico como ferramenta de entrada para soluções interativas. Para atingir o objetivo de baixo custo, foi desenvolvido um tapete sensor de pressão utilizando uma matriz de Velostat, material de resistência variável conforme aplicação de pressão. A leitura do sensor foi realizada por meio de uma placa de desenvolvimento de Arduino Mega e envia os dados via Bluetooth em tempo real. Foram realizados testes de resistência dos sensores e testes do sistema direto no Arduino e com o sistema completo. Foi avaliada a resposta dos sensores, incluindo a sensibilidade do sensor por meio de aumento gradual da carga aplicada. $O$ protótipo desenvolvido mostrou-se ser uma alternativa interessante e de baixo custo para aplicações interativas por meio da pressão dos pés.
\end{abstract}

Index Terms-Sensor de pressão, Interação HumanoComputador, Velostat.

Abstract-Different types of sensors and technologies have been explored to allow human-computer interaction. The search for sensors that allow a natural interaction, without the need for connections affixed to the body, or learning specific commands, is increasing. In this context, this work presents an input acquisition technology for computational systems through pressure, more specifically, pressure sensors in the form of a mat. The objective of this work is to develop and validate a low-cost pressure monitoring system prototype. It is a proof of concept study to present the use of an alternative generic pressure mat as an input tool for interactive solutions. A pressure mat was developed using a Velostat matrix, a material of variable resistance according to pressure application. The sensor data was received by an Arduino Mega development board and sent via Bluetooth in real time. Tests evaluated sensors resistance directly from Arduino and also from the complete integrated system. The sensors' responses were evaluated, including their sensitivity, by gradually increasing the applied load. The prototype developed proved to be an exciting and low-cost alternative for interactive applications through foot pressure.

Keywords-Pressure mat, Human Computer Interaction, Velostat.

\section{INTRODUÇÃO}

A área de HCI (Human Computer Interaction) está relacionada com o projeto, avaliação e implementação de sistemas computacionais interativos. Assim como os humanos comunicam-se entre si através de gestos, fala, expressões faciais, é natural pensar em utilizar os mesmos modos de comunicação entre homem e máquina. Sistemas que não dependem de entrada apenas através de teclados ou joysticks, mas também de dispositivos não tão convencionais, merecem destaque [1]. Este trabalho tem como foco a aquisição de entrada para sistemas computacionais através da pressão, mais especificamente sensores de pressão em forma de tapetes. Uma vantagem desse tipo de sensor é que o usuário fica livre para realizar outros gestos que dependam de diferentes partes do corpo, como mãos e cabeça.

Diferentes tipos de sensores têm explorado esse tipo de interação. O trabalho de Rangarajan et al. [2] realiza uma comparação entre diferentes sistemas de sensoriamento de pressão.

As aplicações interativas por meio de pressões do pé abrange diferentes áreas de atuação, como, por exemplo, os jogos interativos de dança [3]. Na área de reabilitação esse tipo de interação tem ampla aplicação para os exercícios de equilíbrio, por isso é bastante utilizado na reabilitação de pacientes, principalmente neurológico. Para essa aplicação, até o momento, o balance board do Nintendo Wii tem maior destaque como dispositivo interativo [4] [5]. Na área de reconhecimento de gestos, o uso desse tipo de sensores pode ajudar a diferenciar gestos com poses corporais similares, porém com diferentes distribuições de peso [2].

Com o objetivo de usar esse tipo de sensor em HCI, o sensor deve atender a algumas características, que devem ser avaliadas e especificadas para cada aplicação como, por exemplo:

- Pressão máxima suportada;

- Densidade dos sensores capazes de detectar a pressão (número de pontos distintos na matriz);

- Velocidade de leitura da matriz de sensores;

- Acesso aos dados. 
O objetivo deste trabalho é desenvolver um protótipo de um sistema de monitoramento de pressão capaz de provar conceitualmente uma alternativa de baixo custo de tapete de sensores de uso genérico para HCI.

Os objetivos específicos deste projeto são:

- Apresentar uma alternativa de baixo custo frente à implementação de unidades de sensores piezoresistivos com resolução suficiente para indicar regiões do corpo sob pressão;

- Disponibilizar os resultados por meio de uma interface amigável para o usuário;

- Disponibilizar os resultados em tempo real remotamente por meio de comunicação sem fio.

Este artigo está estruturado confome segue: a seção 2 detalha o desenvolvimento do projeto, tanto do ponto de vista do hardware como do software. A seção 3 descreve o processo de validação e testes utilizado para comprovar a corretude do funcionamento do dispositivo proposto. Por fim, a seção 4 destaca pontos importantes sobre trabalho e aponta possíveis melhorias ao projeto.

\section{Desenvolvimento}

A solução para monitorar a pressão sobre o leito de pacientes acamados foi modelada de acordo com os objetivos propostos durante a definição do escopo do trabalho, mas devido à complexidade da produção de um produto completo de ponta a ponta, este desenvolvimento ficará limitado à prova de conceito. Dessa forma, a solução será desenvolvida de modo que justifique uma futura dedicação ao produto comercializável de baixo custo, comparado a outros produtos já comercializados mas que não têm força para entrar no mercado brasileiro.

Devido à aplicação desses sensores em um contexto em que a ergonomia e a robustez são primordiais, é preferível a utilização de sensores de materiais flexíveis para que o sensor não se torne mais um ponto de pressão no corpo do paciente e o uso de sensores mais sensíveis, conferindo confiabilidade e usabilidade deste artefato inteligente. Por esses motivos, a seleção de materiais está voltada para as possibilidades de materiais flexíveis a fim de manter o conforto do paciente, de modo que esse sistema anexo ao leito seja transparente a ele.

De forma compilada, pelo que foi discutido até este ponto, as premissas para a construção do protótipo são: seleção de materiais que promovam a redução de custo sem comprometer a funcionalidade principal do sistema e que sejam viáveis quanto à usabilidade, visto que não podem intensificar a pressão sobre a pele do paciente; seleção de componentes que viabilizem a prototipação em tempo reduzido, suficientes para a prova de conceito, conforme a definição dos objetivos.

\section{A. Tapete de pressão}

Para o tapete de pressão a ideia inicial era realizar a prova de conceito utilizando sensores piezoresistivos. Sensores para a leitura de pressão existem no mercado em variados preços, no entanto, mesmo o mais barato entre eles pode significar um alto preço no produto final, visto a necessidade de milhares de sensores para garantir a qualidade e robustez da leitura da pressão no leito. Por esse motivo, seguiu-se a busca por outros materiais que poderiam ser utilizados na solução proposta.

Além dos sensores, existem materiais com propriedades elétricas que permitem seu uso de forma semelhante ao dos sensores em que valores resistivos do material modificam localmente com a pressão, de modo que é possível mapear os pontos sob pressão nesse tipo de material quando é implementado junto a um circuito, a exemplo do Velostat.

Por esse motivo unidades de sensores piezoresistivos foram substituídas pelo Velostat, material de resistência variável conforme a aplicação de pressão, implementada com fitas condutivas formando as trilhas do circuito, dispostas de maneira que os pontos de interseção entre trilhas de fitas horizontais e verticais formem pontos de leitura. Cada interseção substitui um sensor comercial que seria necessário comprar para implementar o monitoramento da pressão.

Para implementar o circuito sobre o Velostat, utilizou-se as fitas adesivas de cobre interfaceando o Velostat. A razão do cobre ser utilizado nesse desenvolvimento justifica-se pelas suas propriedades condutivas e flexíveis. Para mapear a pressão ao longo de toda a folha do Velostat, é necessário identificar os pontos de leitura, por isso o procedimento de montagem da matriz de sensores consistiu em adesivar fitas condutivas alternadas com fita dupla face em um tecido plástico, posicionadas horizontalmente em uma folha e verticalmente em outra, conforme indicado na Figura 1.

Em seguida foi feito um sanduíche com as folhas de plástico com as fitas horizontais, o Velostat e a outra folha de plástico com fitas verticais, fixadas pelas fitas dupla face. A disposição das fitas após montar o sanduíche das folhas, ilustrado na Figura 1, é importante para que cada interseção corresponda a um sensor comercial, ou seja, o ponto de encontro das fitas perpendiculares são pontos de detecção de pressão. Partindo das dimensões do Velostat, uma folha de $31,5 \mathrm{~cm}$ x $31,5 \mathrm{~cm}$, em que as fitas adesivas de cobre possuem $1 \mathrm{~cm}$ de largura e a fita dupla face $1,2 \mathrm{~cm}$, obteve-se uma matriz com 13 linhas e 13 colunas, totalizando 169 sensores, ou seja, 169 pontos de leitura.

\section{B. Sistema de leitura e transmissão}

Para realizar a leitura da matriz contida no tapete, utilizouse a placa de desenvolvimento Arduino, pois é uma plataforma com uma comunidade grande de desenvolvedores e, portanto, possui bastante documentação disponível. Além disso, é uma plataforma facilmente integrável a outros módulos como Wifi e Bluetooth, que podem ser utilizados para transmitir as informações do leito para um acompanhante que esteja fisicamente distante.

Para a implementação neste projeto, ficou definido que shift registers seriam substituídos pela alimentação fornecida pelos pinos digitais, cuja lógica para a alimentação da matriz ficaria definida em código, e a leitura seria realizada por pinos analógicos no lugar de utilizar multiplexadores devido ao número reduzido de pontos de leitura do protótipo. $\mathrm{O}$ 


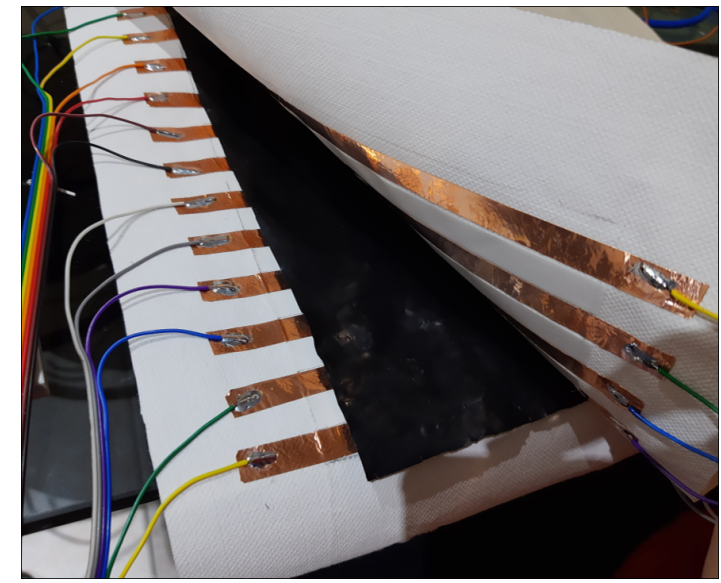

Figura 1. Montagem das folhas com as fitas adesivas e o Velostat.

modelo que mais se adequa à solução é o Arduino Mega 2560, que utiliza o microcontrolador ATmega2560, pois essa versão possui capacidades suficientes para este desenvolvimento, principalmente, quanto à disponibilidade de pinos, já que serão necessários 13 pinos de alimentação e 13 pinos de leitura.

O Arduino Due é uma poderosa placa para grandes projetos e custa o mesmo valor que o Arduino Mega. No entanto, o número máximo de pinos analógicos para entrada é $12 \mathrm{e}$ baseado na dimensão da folha Velostat e da fita de cobre, foram utilizadas 13 trilhas, portanto são necessários 13 pinos. Dessa forma, o Mega tem capacidade tanto operacional para a implementação desse projeto quanto a capacidade física da disposição dos pinos. É sabido que para esse tipo de aplicação podem ser utilizados outros circuitos integrados que diminuam a quantidade de pinos utilizados, como o shift register $\mathrm{e}$ o multiplexador, mas, como mencionado anteriormente, a premissa é a prova de conceito e, por isso, foram realizadas algumas simplificações.

O conversor AD do microcontrolador ATmega2560 possui 10 bits de resolução, ou seja, 1024 valores representarão a intensidade de pressão aplicada no leito. Visto que serão realizadas 13 leituras para cada coluna que receber a alimentação, uma varredura totalizará 169 leituras. Como cada leitura está prevista para 100 microsegundos, conclui-se que uma varredura levará 16900 microsegundos, portanto, em um segundo de leitura contínua, serão realizadas 59 varreduras:

$$
V=\frac{1 s}{\frac{16900 \mu s}{1 \text { varredura }}} \approx 59 \text { varreduras }
$$

$\mathrm{Na}$ definição do diagrama do circuito, os pinos analógicos estão conectados a um resistor pull down para garantir que esses pinos fiquem em um estado conhecido, a $0 \mathrm{~V}$, quando o circuito não estiver recebendo alimentação.

A composição do tapete, o resistor pull down e o Arduino Mega formam o módulo de leitura de pressão do sistema de monitoramento, mas para que essas informações sejam fornecidas a um usuário de forma remota, é preciso desenvolver o módulo de comunicação sem fio. Entende-se que o alcance para receber a informação precisa ser sem limitações e, por isso, a implementação com um servidor Web seria necessária para o produto final, mas para simular a utilização do sistema de monitoramento, utilizou-se o módulo Bluetooth.

Para conectar o Bluetooth ao Arduino e estabelecer a comunicação serial, utilizou-se o pino de $5 \mathrm{~V}$ e GND do Arduino, o RX do ATmega conectado ao TX do Bluetooth por meio de um divisor de tensão e o TX do ATmega ao RX do Arduino diretamente. Dessa forma, pode-se entender que cada tapete será conectado a um hardware, com capacidade para realizar a leitura e enviar as informações. Será utilizado um aplicativo para receber as informações do Bluetooth e apresentar o mapeamento da pressão do leito de forma amigável para o usuário. $\mathrm{O}$ aplicativo desenvolvido é um demonstrativo da comunicação em tempo real, podendo as aplicações serem diversas com a informação provida, desde soluções de HCI como também sistemas de avaliação da pisada do pé, por exemplo.

Tabela I

CUSTO DO PROTÓTIPO

\begin{tabular}{lll}
\hline Item & Quantidade & Valor $(\mathrm{R} \$)$ \\
\hline Arduino Mega + cabo USB & 1 & 120,00 \\
Barramentos macho e fêmea & 4 & 9,00 \\
Bluetooth & $1 \mathrm{~m}$ & 30,00 \\
Cabo Flat & $3 \mathrm{~m}$ & 54,00 \\
Fitas de cobre & $10 \mathrm{~m}$ & 90,00 \\
Fitas dupla face & $10 \mathrm{~m}$ & 12,00 \\
Folhas de plástico & $1 \mathrm{~m}$ & 10,00 \\
Fonte de alimentação & 1 & 15,00 \\
Jumpers & 10 & 5,00 \\
Placa universal de fenolite & 1 & 20,00 \\
Resistores & 16 & 2,00 \\
Velostat & 1 & 108,00 \\
TOTAL & & 475,00 \\
\hline
\end{tabular}

\section{Código do microcontrolador}

No código construído na IDE do Arduino, inicialmente, algumas variáveis armazenam o tempo corrente para ter o ponto de início e fim de uma leitura a fim de manter o período de um ciclo de leituras aproximadamente constante. Além disso, os resultados só são enviados quando o Arduino receber a primeira requisição do aplicativo, por isso há a verificação da variável sending.

A leitura da pressão no tapete é definida em código na função monitora(), na qual duas estruturas de loop concatenadas realizam a alimentação de um pino digital e em seguida faz-se a leitura de todos os pinos analógicos em sequência, voltando para o for mais externo, definindo a alimentação do próximo pino, enquanto todas as outras entradas digitais estão em zero de tensão e realiza novamente a leitura de todos os pinos analógicos até que toda a matriz seja lida.

Vários ciclos de leitura são realizados em milésimos de segundo e, por isso, a cada leitura realizada soma-se o resultado a uma variável para que depois de um tempo prédeterminado seja calculada a média das leituras, conforme a função calculamedia(), que recebe o somatório de cada 
ponto da matriz e divide pela quantidade de leituras realizadas, armazenada em uma variável global.

Para apresentar as leituras em uma escala de 3 valores, a função compara() é chamada para verificar em qual dos intervalos a leitura se encaixa e, consequentemente, temse o retorno da classificação da intensidade da pressão. Esse resultado obtido é enviado via Bluetooth por meio da comunicação serial estabelecida na função imprimeChar(), para um dispositivo em que será visualizado o resultado em escala de cores. Cada resultado da classificação possui uma cor representativa em tons de verde, quanto mais clara a cor, mais intensa é a pressão, conforme definido no código da aplicação detalhado na próxima seção.

\section{Código do aplicativo}

No App Inventor a aplicação foi projetada para se conectar com o módulo de monitoramento e atribuir cores às classificações obtidas na função compara(). Para realizar a conexão, escaneia-se os dispositivos Bluetooth disponíveis, o usuário seleciona o HC-06 e insere a senha. Utilizou-se o caractere limitador $\mathrm{X}$, indicado por 88 , segundo a tabela ASCII, para que toda vez que o $\mathrm{X}$ for recebido todas as informações anteriores recebidas sejam apresentadas em tela. O envio do texto " 1 " para o Arduino indica que ele já pode enviar as leituras serialmente, ou seja, trata-se de uma chamada de requisição.

Assim, enquanto o Bluetooth estiver conectado, a variável tam recebe o tamanho de bytes disponíveis e, então, os dados recebidos pela serial preenchem a variável valores, que terá exatamente a leitura de uma matriz completa. Em seguida, uma lista é criada com esses valores, a partir da separação dos caracteres pelos espaços que também foram enviados; se o tamanho da lista for maior ou igual a 169, o que corresponde à quantidade de pontos de leitura, então trata-se cada caractere atribuindo uma cor correspondente.

Para obter a posição na lista, foi utilizada a expressão i*13 + j-13 e não mais um for concatenado como foi utilizado na IDE do Arduino. Cada caractere da lista será apresentado na forma de matriz e, definidas as coordenadas, uma linha com espessura será desenhada, formando um quadrado com a cor definida anteriormente.

\section{VALIDACÕES E TESTES}

Para conhecer mais das propriedades do material, realizouse alguns testes iniciais antes de implementar o sistema de monitoramento. O primeiro deles foi realizar a medição da resistência em pontos aleatórios da folha Velostat, utilizando o multímetro Minipa ET-1000, no qual a resistência obtida foi na ordem de $40 \mathrm{k} \Omega$, portanto, pode-se concluir que entre as fitas de cobre haverá um resistência de $40 \mathrm{k} \Omega$.

$\mathrm{Na}$ configuração em que as fitas de cobre foram adesivadas no próprio Velostat, a resistência variou bastante entre cada ponto, devido à presença do material adesivo entre a parte metálica e o Velostat, mas ainda assim possui uma resistência menor, entre $3 \mathrm{k} \Omega$ e $10 \mathrm{k} \Omega$.
$\mathrm{Na}$ versão final, em que as fitas de cobre estão adesivadas em uma folha de plástico formando um sanduíche com o Velostat, no qual a parte metálica do cobre está diretamente em contato com o material resistivo, a resistência fica mais uniforme entre os pontos, próximo de $1,2 \mathrm{k} \Omega$. Junto a essa resistência, foi associado um resistor pull down de resistência 1,2 $\mathrm{k} \Omega$ ligado ao terra, para garantir que não haverá estados desconhecidos nos pinos analógicos. Quando os sensores são pressionados, a resistência variável reduz, aumentando o nível de tensão lido nos pinos analógicos.

Com o sistema de monitoramento finalizado, iniciou-se os testes de validação. No primeiro deles, utilizou-se o terminal serial da IDE do Arduino para visualizar os resultados da leitura em números, ou seja, ainda não se utilizou o programa final que faz uma classificação das leituras em três grupos de intensidade. Em um segundo momento, utilizou-se todo o sistema.

A primeira fase de testes contou com três experimentos: verificar em cada sensor a resposta para pressões provocadas por aproximadamente $1 \mathrm{~kg}$, obter o intervalo de leitura para cada classificação que será apresentada no aplicativo e obter a leitura de alguns pontos para diferentes pesos conhecidos a fim de obter a relação entre massa e leitura do tapete.

Testando ponto a ponto, identificou-se que a maioria das respostas para mais de $1 \mathrm{~kg}$ é um valor entre 800 e 1000 (valores binários da escala de 0 a 1023 provenientes do conversor analógico-digital de 10 bits), representantes da tensão lida no pino analógico. Alguns poucos apresentam resposta entre 600 e 799 e uma região específica não responde à pressão aplicada. Nessa região foram realizados os primeiros testes, mas devido à fricção à qual a região foi submetida durante a remoção das fitas dupla face e fitas de cobre para fazer a montagem final na folha Velostat, o material foi danificado.

Fazendo testes pressionando com o dedo e em seguida posicionando o menor bloco, Bloco 1, constatou-se que a partir de 87 g é possível concluir que houve aumento da pressão no sensor de forma segura, pois antes desse valor a leitura é quase zero. Além disso, posicionando os Blocos 1 e 2, adicionou-se uma caneca para aos poucos adicionar arroz, acompanhando em qual peso a leitura mudaria para 500, a fim de representar no aplicativo uma cor mais intensa. Esse peso foi de $350 \mathrm{~g}$, dessa forma, representou-se com verde escuro o intervalo de leitura de $80 \mathrm{~g}$ a $349 \mathrm{~g}$ e com uma cor mais intensa o intervalo de leitura de $350 \mathrm{~g}$ a $1 \mathrm{~kg}$.

Utilizou-se o tapete de monitoramento conectada ao Arduino e ao terminal serial; foram selecionados 5 diferentes pesos, combinando 3 corpos de provas cujos pesos são distribuídos uniformemente. Realizou-se o teste em 4 sensores e a leitura foi registrada após 30 segundos para cada ponto e peso, pois observou-se que há um tempo de acomodação do material para estabilizar a resistência. A partir do momento que se coloca um bloco sobre o tapete, a leitura pode aumentar em 100 unidades.

Conforme detalhado na Tabela II, mapearam-se os valores de leitura em função dos pesos 87,66 g, 376,92 g, 650,29 g, $1140,07 \mathrm{~g}$ e 1429,33 g nas posições de linha 1 e coluna 10 , 
linha 1 e coluna 8, linha 6 e coluna 7 , linha 4 e coluna 1 . A partir das médias das leituras em cada ponto e para cada peso, obteve-se a relação entre peso e leitura. Apesar do tema úlceras de pressão estar diretamente relacionado à pressão, a massa foi a grandeza sob observação nesse teste, pois ela é facilmente compreendida dentro do contexto deste projeto, em que pessoas acamadas exercem um determinado peso sobre o leito.

Tabela II

VALORES OBTIDOS DE QUATRO DIFERENTES SENSORES PARA CINCO DIFERENTES PESOS

\begin{tabular}{llllll}
\hline Massa $(\mathrm{g})$ & Leitura 1 & Leitura 2 & Leitura 3 & Leitura 4 & Média \\
\hline 87,65 & 380 & 305 & 390 & 357 & 358 \\
376,92 & 550 & 525 & 585 & 620 & 570 \\
650,29 & 775 & 665 & 684 & 692 & 704 \\
1140,07 & 815 & 718 & 748 & 844 & 781,25 \\
1429,33 & 839 & 800 & 870 & 918 & 856,75 \\
Posição & L1.C10 & L1.C8 & L6.C7 & L4.C1 & \\
\hline
\end{tabular}

Nos testes, o Bloco 1 foi utilizado como base, pois a sua base é estreita, o que permite realizar o teste para apenas um ponto de leitura. No entanto, a área não é exatamente a mesma, por isso, sabendo que a área do sensor é $55 \%$ da área do Bloco 1 , todos os pesos considerados correspondem a aproximadamente $55 \%$ do peso real dos blocos.

Finalmente, na segunda fase de testes, utilizou-se todo o sistema de monitoramento, o tapete de sensores, o Arduino para realizar a leitura, o Bluetooth para transmitir os dados e o aplicativo para apresentar as regiões sob pressão. A Figura 2 apresenta o resultado para duas diferentes situações de aplicação de peso.

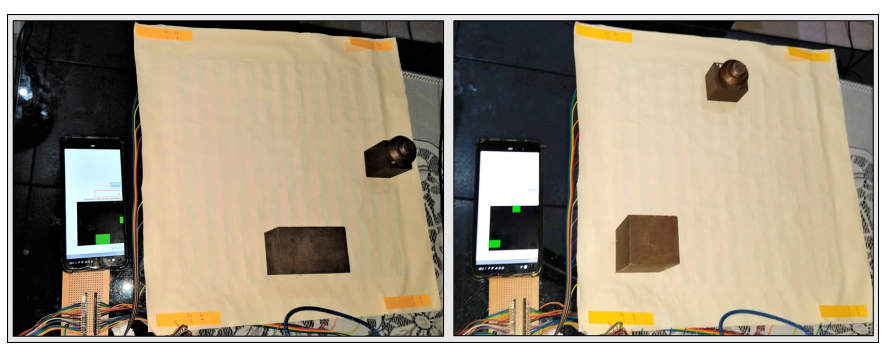

Figura 2. Demonstração do teste de integração por imagem.

\section{CONCLUSÃO}

O protótipo discutido e desenvolvido neste trabalho é uma proposta de um artefato digital que pode gerar impacto não só na área de HCI, mas também em outras áreas. Por exemplo, na área médica, poder-se-ia utilizar o sensor desenvolvido para prevenção de úlceras de pressão em pacientes acamados.

Dado o perfil deste trabalho de provar conceitualmente uma funcionalidade com materiais alternativos para um dispositivo capaz de monitorar a pressão em um tapete, o desenvolvimento realizado abre oportunidades de estudos para a finalização do produto, até que ele apresente características suficientes para ser produzido em larga escala.
Uma das dificuldades apresentadas durante o desenvolvimento foi a estabilidade da leitura, tanto o tapete tem um tempo de acomodação quanto a leitura tem um intervalo de variação limitado para interpretação do resultado em código. Assim, é importante investir em robustez, realizando o tratamento dos dados obtidos na leitura, de forma que os resultados apresentados sejam mais precisos. Como sugestão, poder-se-ia amplificar o sinal obtido no sensor, trazendo um detalhamento maior quanto à escala de pressão ao qual o corpo está submetido, e filtrar o sinal com o propósito de remover ruídos.

Por tratar-se de uma solução interdisciplinar, em que muito da lógica desenvolvida é baseada em conhecimentos de fisiologia, um dos levantamentos que poderia ser realizado é a relação entre a pressão exercida sobre o corpo e o tempo de exposição para aquela pressão em função de cada área do corpo, dentre as com maior proeminência óssea.

Para otimizar o hardware deste projeto, é possível desenvolver uma placa de circuito impresso completamente voltada para a aplicação, sem as redundâncias da placa de desenvolvimento. É interessante que esse projeto seja desenhado para a malha em dimensões reais, portanto, seria necessário implementar com um shift register e um multiplexador, além do amplificador e conversor AD para melhorar a resolução.

É possível também realizar o estudo de viabilidade para reduzir o tamanho dos sensores, aumentando em precisão e resolução.

Após as melhorias que essas pesquisas podem promover, é necessário um estudo de como automatizar a fabricação do tapete proposto, cujo elemento principal é o Velostat, associando aos cálculos de custos, de modo que seja mantida a proposta de baixo custo frente às demais alternativas de mercado.

\section{REFERÊNCIAS}

[1] A. C. S. Medeiros. (2015) Processo de desenvolvimento de gestos para interfaces de usuário. [Online]. Available: https://repositorio.ufpb.br/jspui/bitstream/tede/7863/2/arquivototal.pdf

[2] S. Rangarajan, A. Kidané, G. Qian, and S. Rajko, "Design optimization of pressure sensing floor for multimodal human-computer interaction," in Human computer interaction. IntechOpen, 2008.

[3] L. B. Azevedo, D. B. Watson, C. Haighton, and J. Adams, "The effect of dance mat exergaming systems on physical activity and health-related outcomes in secondary schools: results from a natural experiment," $B M C$ public health, vol. 14, no. 1, p. 951, 2014.

[4] J. Iruthayarajah, A. McIntyre, A. Cotoi, S. Macaluso, and R. Teasell, "The use of virtual reality for balance among individuals with chronic stroke: a systematic review and meta-analysis," Topics in stroke rehabilitation, vol. 24, no. 1, pp. 68-79, 2017.

[5] Y. Laufer, G. Dar, and E. Kodesh, "Does a wii-based exercise program enhance balance control of independently functioning older adults? a systematic review," Clinical interventions in aging, vol. 9, p. 1803, 2014. 